\title{
Stable habituation deficits in the early stage of psychosis: a 2-year follow-up study
}

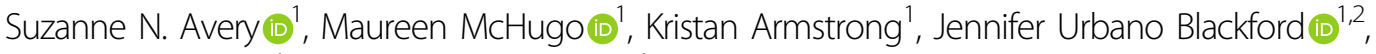

Neil D. Woodward ${ }^{1}$ and Stephan Heckers (1)

\begin{abstract}
Neural habituation, the decrease in brain response to repeated stimuli, is a fundamental, highly conserved mechanism that acts as an essential filter for our complex sensory environment. Convergent evidence indicates neural habituation is disrupted in both early and chronic stages of schizophrenia, with deficits co-occurring in brain regions that show inhibitory dysfunction. As inhibitory deficits have been proposed to contribute to the onset and progression of illness, habituation may be an important treatment target. However, a crucial first step is clarifying whether habituation deficits progress with illness. In the present study, we measured neural habituation in 138 participants (70 early psychosis patients (<2 years of illness), 68 healthy controls), with 108 participants assessed longitudinally at both baseline and 2-year follow-up. At follow-up, all early psychosis patients met criteria for a schizophrenia spectrum disorder (i.e., schizophreniform disorder, schizophrenia, schizoaffective disorder). Habituation slopes (i.e., rate of fMRI signal change) to repeated images were computed for the anterior hippocampus, occipital cortex, and the fusiform face area. Habituation slopes were entered into a linear mixed model to test for effects of group and time by region. We found that early psychosis patients showed habituation deficits relative to healthy control participants across brain regions, and that these deficits were maintained, but did not worsen, over two years. These results suggest a stable period of habituation deficits in the early stage of schizophrenia.
\end{abstract}

\section{Introduction}

Habituation, defined as the decrease in response to repeated stimulus exposures, is an elementary and ubiquitous form of behavioral plasticity ${ }^{1}$. Acting as a sensory 'firewall' ${ }^{2}$, habituation is thought to gate the vast amounts of irrelevant and repeated sensory information experienced in our everyday environment, allowing prioritization of salient and actionable items. Rapid habituation may conserve limited processing resources and act as a building block for normal cognitive function ${ }^{3,4}$. In contrast, failure to rapidly habituate has been proposed to lead to diminished attentional focus ${ }^{5}$, cognitive fragmentation $^{6}$, and inappropriate behavioral responses ${ }^{7}$. Thus, habituation may be a foundation for cognitive

\footnotetext{
Correspondence: Suzanne N. Avery (suzanne.avery@vumc.org)

${ }^{1}$ Department of Psychiatry and Behavioral Sciences, Vanderbilt University Medical Center, Nashville, TN, USA

${ }^{2}$ Research Health Scientist, Research and Development, Department of Veterans Affairs Medical Center, Nashville, TN, USA
}

integrity in schizophrenia wherein even modest impairments in habituation may be capable of producing clinical impairment ${ }^{6}$.

Impairments in habituation and sensory filtering in schizophrenia have been recognized for over a century ${ }^{8}$ and are now considered core features of the illness ${ }^{7,9}$. Habituation deficits have been consistently identified at all levels of measurement, including at the behavioral, electrophysiological, and neural level. Historically, habituation deficits in schizophrenia were identified using wellvalidated measurements such as habituation of eye-blink startle $^{9,10}$ and autonomic nervous system response ${ }^{11,12}$. More recently, studies using electroencephalogram (EEG) and functional magnetic resonance imaging (fMRI) measures have demonstrated neural habituation deficits in schizophrenia, including reduced habituation to auditory evoked potentials ${ }^{13-15}$, and reduced habituation to repeated visual stimuli ${ }^{16-18}$. Although the specific neural processes underlying habituation deficits remain elusive ${ }^{19}$, 
at all levels of measurement, habituation is thought to reflect inhibitory mechanisms of plasticity ${ }^{20}$. Studies in Drosophila have shown that habituation requires effective inhibition of excitatory response via a centralized, network-level potentiation of inhibitory synapses $^{21,22}$. Inhibitory function is disrupted in schizophrenia, and failure of inhibition is among the most compelling explanations for the widely-observed sensory processing impairment seen in patients ${ }^{23}$.

An important brain region associated with inhibitory deficits in schizophrenia is the hippocampus ${ }^{24-26}$. Playing a fundamental role in filtering of incoming sensory information in support of learning, the hippocampus rapidly habituates to repeated visual information ${ }^{27-31}$ in healthy individuals. However, schizophrenia patients show deficits in hippocampal habituation ${ }^{16-18}$ and inhibitory function ${ }^{24}$ to repeated visual stimuli, with inhibitory deficits linked to impaired information processing ${ }^{32}$. Recent findings convincingly show inhibitory deficits also exist in the visual cortex ${ }^{33,34}$ in schizophrenia. Repeated visual stimuli elicit robust habituation in the visual cortex $^{35}$ and fusiform gyrus ${ }^{35,36}$ in healthy individuals, while schizophrenia patients show habituation deficits in both regions ${ }^{16,17}$. Together, these findings suggest correspondence between habituation and inhibitory function in the hippocampus and ventral visual stream, in-line with evidence in the non-human literature that implicates habituation as a deficit of inhibition.

Disruption of inhibitory function may be involved in the pathogenesis of schizophrenia ${ }^{37}$. In recent years, there has been growing interest in defining neural mechanisms that could be used as treatment targets for interventions that may attenuate progression of illness. The early phase of psychotic illness has been proposed as a critical period during which deterioration progresses rapidly and interventions may be most impactful ${ }^{38}$. Habituation, first described in Aplysia ${ }^{39}$, is a fundamental process that is highly conserved across species, making it an ideal mechanism for translational study. However, understanding whether habituation deficits progress with illness is a crucial first step. Although neural habituation deficits have been observed in both early ${ }^{17}$ and chronic stages of schizophrenia ${ }^{16,18}$, differences in task administration and analysis techniques across studies have made it difficult to determine whether habituation deficits differ by illness stage.

We previously identified habituation deficits in three regions-the anterior hippocampus, occipital pole, and fusiform face area (FFA) - in a cohort of early psychosis patients with less than 2 years of illness ${ }^{17}$. The aim of the current study was to determine whether these deficits progressed over the next two years of illness. Participants who completed the cohort study were asked to return for a follow-up visit 2 years later. During the follow-up visit, participants repeated the visual-stimulus repetition task administered at baseline using an alternate image set. This is the first longitudinal analysis of the repetition task. We hypothesized that habituation deficits progress over two years of illness.

\section{Methods and material \\ Participants}

One-hundred thirty-eight participants (70 early psychosis patients and 68 healthy control participants) were enrolled in a prospective longitudinal study of habituation between May 2013 and November 2017 and followed for two years (Supplementary Figs. 1 and 2). Cross-sectional baseline data from some of our participants have already been reported ${ }^{17}$ (62 early psychosis patients and 68 healthy control participants); this is the first report of follow-up data.

All participants were assessed by a trained rater using the Structured Clinical Interview for the DSM-IV (SCID I-P) ${ }^{40}$ and diagnoses were confirmed by a senior psychiatrist (S.H.). Patients in the early stage of a psychotic disorder were recruited from the outpatient clinics and inpatient units of Vanderbilt Psychiatric Hospital. Inclusion criteria included: (1) age 13-40; (2) premorbid IQ above 75 ; (3) <2 years of psychotic illness; and (4) meeting criteria A for schizophrenia ${ }^{40,41}$. Early psychosis participants were excluded if they reported active substance use or dependence in the past month or if a psychotic disorder due to a medical condition was diagnosed. Additionally, only patients meeting criteria for a schizophrenia spectrum disorder (schizophreniform disorder, schizoaffective disorder, or schizophrenia) at 2-year follow-up were included in this analysis. Patients were assessed for current mood, psychotic symptom severity, and antipsychotic medication dose at baseline and two-year follow-up (see Supplementary Methods). More than $85 \%$ of the patient sample were treated with antipsychotic medication at the time of the study.

Healthy control participants were recruited from the community via advertisement. Healthy control participants were excluded if they met criteria for any Axis I disorder $^{40,41}$ at enrollment and at the end of the study, had a first-degree relative with a psychotic illness, or had current psychotropic medication use. Groups were recruited with the goal to match for mean age, gender, race, and parental education (Table 1).

All participants provided written informed consent and received monetary compensation for their time. The study was approved by the Vanderbilt University Institutional Review Board, Nashville, TN.

\section{Clinical assessment and measures}

We collected clinical data at enrollment and two-year follow-up to characterize trajectory of illness. The full 
Table 1 Demographics.

\begin{tabular}{|c|c|c|c|c|c|c|}
\hline \multirow[t]{2}{*}{ Demographics } & \multicolumn{2}{|c|}{ Healthy control, $n=68$} & \multirow{2}{*}{\multicolumn{2}{|c|}{ Early psychosis, $n=70$}} & \multicolumn{2}{|c|}{$\begin{array}{l}\text { Healthy control vs. early } \\
\text { psychosis }\end{array}$} \\
\hline & \multicolumn{2}{|c|}{ Mean \pm SD } & & & Statistic & $p$ \\
\hline Age at enrollment, years & \multicolumn{2}{|l|}{$22 \pm 2.9$} & \multicolumn{2}{|l|}{$21 \pm 3.8$} & $F_{1,137}=0.22$ & 0.64 \\
\hline Sex, $\%$ male & \multicolumn{2}{|l|}{$72 \%$} & \multicolumn{2}{|l|}{$77 \%$} & $X^{2}=0.47$ & 0.49 \\
\hline Race, white/black/other & \multicolumn{2}{|l|}{$53 / 11 / 4$} & \multicolumn{2}{|l|}{$53 / 16 / 1$} & $x_{2}^{2}=2.70$ & 0.26 \\
\hline Handedness, \% right & \multicolumn{2}{|l|}{$91 \%$} & \multicolumn{2}{|l|}{$94 \%$} & $x^{2}=0.50$ & 0.48 \\
\hline Premorbid IQ, WTAR & \multicolumn{2}{|l|}{$112 \pm 10.5$} & \multicolumn{2}{|l|}{$103 \pm 16.0$} & $F_{1,129}=14.66^{\mathrm{a}}$ & $<0.001^{*}$ \\
\hline Participant's education, years & \multicolumn{2}{|l|}{$15 \pm 1.9$} & \multicolumn{2}{|l|}{$13 \pm 2.3$} & $F_{1,137}=10.79$ & $<0.001^{*}$ \\
\hline Parental education, years & \multicolumn{2}{|l|}{$15 \pm 2.4$} & \multicolumn{2}{|l|}{$15 \pm 2.7$} & $F_{1,137}=0.36$ & 0.55 \\
\hline \multirow[t]{2}{*}{ Clinical characteristics } & \multicolumn{2}{|c|}{ Baseline, $n=64$} & \multicolumn{2}{|c|}{ Follow-up, $n=59$} & \multicolumn{2}{|c|}{ Baseline vs. follow-up } \\
\hline & Mean \pm SD & Range & Mean \pm SD & Range & Statistic & $p$ \\
\hline Diagnosis, SZF/SZ/BP & $42 / 19 / 3$ & - & $16 / 43 / 0$ & - & - & - \\
\hline Duration of illness, months & $7 \pm 5.7$ & $1-24$ & $31 \pm 5.5$ & $24-48$ & - & - \\
\hline $\mathrm{CPZ}, \mathrm{mg}$ & $271 \pm 181.3$ & $0-750$ & $196 \pm 219.7$ & 0-900 & $F_{1,122}=4.34$ & $0.04^{*}$ \\
\hline Antipsychotic treatment, \% & $84 \%$ & - & $64 \%$ & - & $x^{2}=6.49$ & $0.01^{*}$ \\
\hline PANSS Total & $66 \pm 20.1$ & $34-114$ & $51 \pm 14.9$ & $33-104$ & $\mathrm{~F}_{1,122}=22.52$ & $<0.001^{*}$ \\
\hline PANSS-Positive & $17 \pm 6.9$ & $7-36$ & $13 \pm 4.6$ & $7-27$ & $F_{1,122}=13.40$ & $<0.001^{*}$ \\
\hline PANSS-Negative & $17 \pm 7.8$ & $7-37$ & $12 \pm 6.3$ & $7-32$ & $F_{1,122}=14.83$ & $<0.001^{*}$ \\
\hline PANSS-General & $33 \pm 9.1$ & $17-59$ & $26 \pm 7.0$ & $17-46$ & $F_{1,122}=18.77$ & $<0.001^{*}$ \\
\hline HAM-D & $9 \pm 6.3$ & $0-24$ & $5 \pm 4.2$ & $0-15$ & $\mathrm{~F}_{1,122}=14.94$ & $<0.001^{*}$ \\
\hline YMRS & $3 \pm 4.4$ & $0-20$ & $2 \pm 2.7$ & $0-15$ & $F_{1,121}=1.18^{b}$ & 0.28 \\
\hline
\end{tabular}

WTAR Wechsler Test of Adult Reading, SZF schizophreniform, SZ schizophrenia, BP bipolar disorder with psychotic features, CPZ chlorpromazine equivalent, PANSS Positive and Negative Syndrome Scale, HAM-D Hamilton Depression Rating Scale-17 item, YMRS Young Mania Rating Scale. Asterisk $\left({ }^{*}\right)$ denotes significant $p$ values $(p \leq 0.05)$.

aWTAR scores are reported for native English speakers (early psychosis, $n=68$; healthy control, $n=63$ ).

${ }^{b}$ One patient missing YMRS at baseline.

clinical assessment has been previously detailed ${ }^{42}$. Briefly, early psychosis participants completed in-person clinical interviews, including the SCID ${ }^{43}$, Positive and Negative Syndrome Scale (PANSS) ${ }^{44}$, Depression Rating Scale $(\text { HAM-D })^{45}$, Young Mania Rating Scale (YMRS) ${ }^{46}$, and Screen for Cognitive Impairment in Psychiatry (SCIP) ${ }^{47}$. Duration of psychosis was calculated as the date of onset of psychosis until the date of study enrollment. All data gathered during the in-person interviews were augmented by extensive review of all available medical records. Final diagnoses were made by psychiatrist S.H. during diagnostic consensus meetings.

\section{Experimental paradigm \\ Repetition task}

Participants completed a repetition task designed to elicit habituation to repeated stimuli ${ }^{16,17}$ (Supplementary
Fig. 3). The repetition task included four 2-min runs of a repeated neutral face (run 1 and 2) and a repeated neutral object (table, box of tissues; run 3 and 4). Images were presented for $500 \mathrm{~ms}$ followed by a $500 \mathrm{~ms}$ black screen. To promote and assess attention, a target detection task was included. Targets were small versions of the face or object images $\left(25 \%\right.$ of original size ${ }^{36}$ ) presented on $10 \%$ of trials. No targets were presented during the first $10 \mathrm{~s}$ of stimulus repetitions. Participants were asked to press a button during each small image presentation. Target detection was high (detection means $>93 \%$ ) and similar between groups and across visits $(p s>0.36$; see Supplementary Results).

\section{Imaging data acquisition and processing}

Imaging data were collected on a $3 \mathrm{~T}$ Philips Intera Achieva magnetic resonance imaging (MRI) scanner 
located in the Vanderbilt University Institute for Imaging Science. A high-resolution T1-weighted fast field echo (FFE) structural scan and four 2.5-min functional echo planar images (EPI) were acquired for each subject (see Supplementary Methods for details). Functional data were motion corrected, coregistered to the subject's structural image, and smoothed using a $6 \mathrm{~mm}$ Gaussian kernel in SPM12 (http://www.fil.ion.ucl.ac.uk/spm). The first-level (participant) temporal model was estimated on smoothed, native-space functional data using a general linear model ${ }^{48}$. Small images, outliers, and motion (rotation, translation, relative displacement; see Quality control below) were entered into the first-level general linear model $^{48}$ as regressors of no interest and residuals were written for second-level analysis. No temporal filter was applied because slow signal changes may represent habituation to stimulus category.

\section{Quality control}

Functional images were visually inspected for artifacts and signal dropout. Motion (translation, rotation, and relative displacement) and outliers were computed using Artifact Detection Tools (ART; Neuroimaging Informatics Tools and Resources Clearinghouse (NITRC)). Outlier volumes were defined as volumes with global intensity change $>5 \mathrm{SD}$ from mean, or motion $>3 \mathrm{~mm}$. Functional data with $\geq 2$ outlier volumes within the $10 \mathrm{~s}$ habituation window (see Habituation below) were excluded. One patient had 2 outlier volumes at follow-up and was removed from follow-up analysis. The majority of participants had no outliers at either study visit (baseline, $0 \%$; follow-up, $1 \%$; $p s \geq 0.41$ ). Motion was low and similar between groups ( $p s \geq 0.16$; Supplementary Table 1).

\section{Data analysis \\ Regions of interest (ROIs)}

Our goal was to test whether previously identified habituation deficits ${ }^{17}$ in the anterior hippocampus, occipital pole, and fusiform face area (FFA) were maintained over 2-year follow-up. Therefore, ROIs were delineated the same way as in our prior cohort analysis ${ }^{17}$. Subjectspecific hippocampal and occipital pole masks were created using in-house automated multiatlas segmentation techniques ${ }^{49,50}$. Separate baseline and follow-up ROIs were created to account for potential changes in gray matter volume. Hippocampal masks were manually segmented at the uncal apex ${ }^{51,52}$ by a trained rater (S.A.) to create anterior hippocampal ROIs. The FFA was defined in each subject using a localizer task ${ }^{53}$ (Supplementary Methods). Although the FFA was originally proposed as a face-selective region, its role has since been understood as one involving visual expertise $e^{54,55}$. Because our stimuli were common objects (table, box of tissues) we expected FFA involvement in processing. Three healthy control participants ( 2 at baseline, 1 at follow-up) and six patients (all at follow-up) were excluded from FFA analyses because: the localizer scan was not collected due to scanning time constraints (healthy control $=2$, early psychosis $=4$ ); or no FFA clusters were identified (healthy control $=1$, early psychosis $=2$ ).

\section{Habituation}

Habituation was defined as the slope of change in neural signal over repeated stimulus presentations. Consistent with our prior cohort analysis ${ }^{17}$, the habituation window was defined as the window between novelty response and the return to baseline in the healthy control group. Novelty response was defined as the peak signal magnitude following the initial presentation of a stimulus. To minimize confounding effects of repeated exposure to the scanner environment, the habituation window was calculated from the baseline visit. Healthy control signal peaked $8 \mathrm{~s}$ following stimulus onset and returned to baseline by $18 \mathrm{~s}$ following stimulus onset, resulting in a $10 \mathrm{~s}$ habituation window. This window is consistent with prior fMRI studies of habituation in healthy participants ${ }^{56}$; additionally, this window did not include any small image presentations to minimize the effects of small images on habituation results ${ }^{57}$. Novelty response was defined as the fMRI signal amplitude at the beginning of the habituation window. As our prior cohort analysis ${ }^{17}$ revealed between-group differences in habituation to objects, we analyzed the repeated objects run here. For consistency with prior analyses ${ }^{16,17}$ the first objects run was used for habituation analyses, as neural response is minimal following initial stimulus exposure.

Average timeseries for each ROI was extracted from participants' residual time course data using MarsBar ${ }^{58}$. Residual time courses were plotted by hemisphere for each subject and calculations were conducted using Matlab R2017b (The MathWorks Inc., Natick, MA). Signal in the left and right hemispheres were highly correlated across regions; to increase statistical power and minimize type I error, data were averaged across hemispheres.

\section{Habituation slope}

Habituation is highly dependent on the magnitude of the novelty response-that is, there is more opportunity for signal to attenuate over time if signal is initially high. Because we were interested in examining differences in rate of habituation independent of differences in novelty response, we calculated a normalized habituation slope $\left(b^{\prime}\right)$, corrected for novelty response differences, for each participant $^{59-61}$. Habituation slope $\left(b^{\prime}\right)$ values were calculated for each participant using linear regression analysis (see Supplementary Methods for details). Fig. 1 summarizes the habituation slope analysis. 


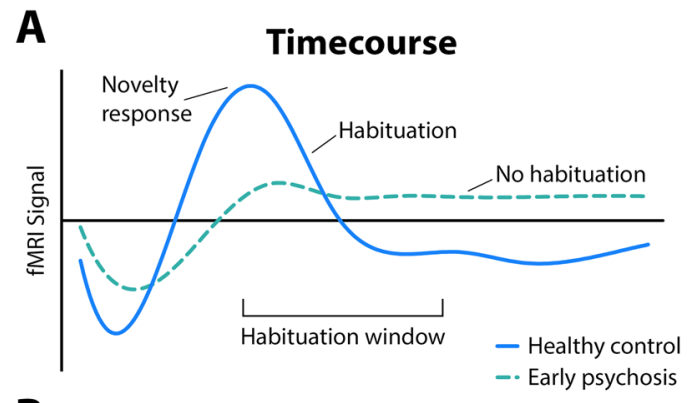

B Habituation Slope

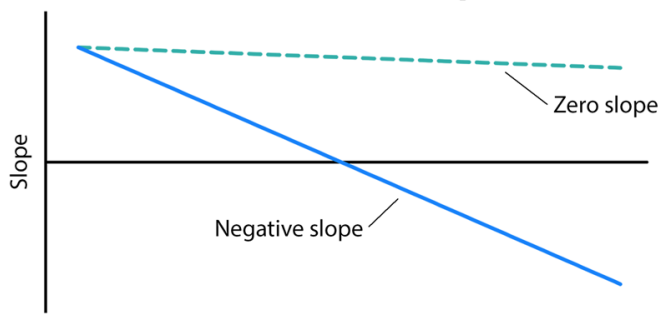

Fig. 1 We studied habituation of neural response over time.

Functional magnetic resonance imaging (fMRI) signal was extracted for a $10 \mathrm{~s}$ window beginning with the novelty response $(\mathbf{A})$ and

habituation slopes were calculated (B). Habituation was characterized as the change in fMRI signal over time with more negative slopes

indicating greater habituation.

\section{Statistical analysis}

Linear mixed effects models tested for habituation rate differences by group and time, with group included as a fixed factor, time as a repeated factor, and participant as a random factor. Because habituation deficits within each region were previously identified in an overlapping sample $^{17}$, regions were tested separately for time and group effects. Follow-up within-group one-sample $t$-tests tested whether habituation slopes were significantly less than zero (h0 $=0$, no habituation; $p \leq 0.05$ ). Cohen's $d$ effect sizes were calculated using the formula contained in ref. ${ }^{62}$. Before completing the study, we conducting a power analysis and determined that with the present sample size, we would have $80 \%$ power to detect an effect size of $d=0.47$ in our primary longitudinal analysis. Spearman correlations tested for associations between habituation and cognitive and cognitive measures. Spearman correlations test for monotonic rather than linear relationships between variables. Spearman correlations were corrected for multiple comparisons across measures within group, time, and region (FWE-adjusted $p \leq 0.05)$. Statistical analyses were performed using SAS software v9.4 (SAS Institute Inc., Cary, NC).

\section{Results}

\section{Clinical sample}

The patient sample was young (mean age 21 years), primarily male (77\%), Caucasian (76\%), and educated (13 years education; Table 1$)$. The majority $(47,67 \%)$ were diagnosed with schizophrenia or schizoaffective disorder after two years of follow-up. Within this group, 17 already met criteria at study entry, 26 progressed from an initial diagnosis of schizophreniform disorder, and 4 converted from an initial diagnosis of psychotic bipolar disorder. A minority $(17,24 \%)$ were diagnosed with schizophreniform disorder at study entry and did not progress to another diagnosis within two years. Early psychosis patients had lower clinical symptoms and $\mathrm{CPZ}$ equivalent dose at follow-up than at baseline (Table 1).

\section{Exclusions and attrition}

Of the 138 participants, data at either baseline or followup for 16 participants were excluded due to: low data quality at baseline (six patients, one healthy control) or follow-up (three patients, three healthy controls; see Quality control); or an ineligible diagnosis at follow-up (two patients, one healthy control; see Supplementary Methods for full details). Additionally, 6 early psychosis patients and 12 healthy control participants were unavailable to return for the follow-up visit. This resulted in an analysis group of 104 longitudinal participants (53 patients, 51 healthy controls), and an additional 34 participants with baseline (11 patients, 16 healthy controls) or follow-up data (6 patients, 1 healthy control; Supplementary Figs. 1 and 2). The 6 early psychosis participants without a 2-year follow-up visit had been diagnosed with schizophrenia $(n=2)$ or schizophreniform disorder $(n=4)$ at study entry and were not demographically and clinically different from early psychosis participants who completed both visits ( $p$ 's $\geq 0.53)$.

\section{Novelty response}

Novelty responses were similar between groups (Supplementary Table 2) and were significantly greater than baseline across regions (Supplementary Table 3).

\section{Habituation}

Habituation slopes at baseline and 2-year follow-up are presented in Fig. 2. We found a main effect of group in each region (anterior hippocampus, $F_{1,136}=11.92$, $\beta=0.11, \mathrm{SE}=0.03$, effect size $=0.57, p=0.001$; occipital pole, $F_{1,136}=9.91, \beta=0.14, \mathrm{SE}=0.05$, effect size $=0.51, p=0.002$; FFA, $F_{1,136}=12.52, \beta=0.16$, $\mathrm{SE}=0.05$, effect size $=0.58, p=0.001$ ), with early psychosis patients habituating $\sim 11-16 \%$ slower than healthy control participants across regions (anterior hippocampus $=11 \%$, occipital pole $=14 \%$, FFA $=16 \%$ ). The FFA showed a trend effect of time $\left(F_{1,93}=3.78\right.$, $\beta=-0.08, \mathrm{SE}=0.04$, effect size $=0.35, p=0.055$ ), with less habituation in both groups at follow-up compared to baseline (no group by time interaction, $p=0.58$ ). Neither the anterior hippocampus nor the occipital pole 

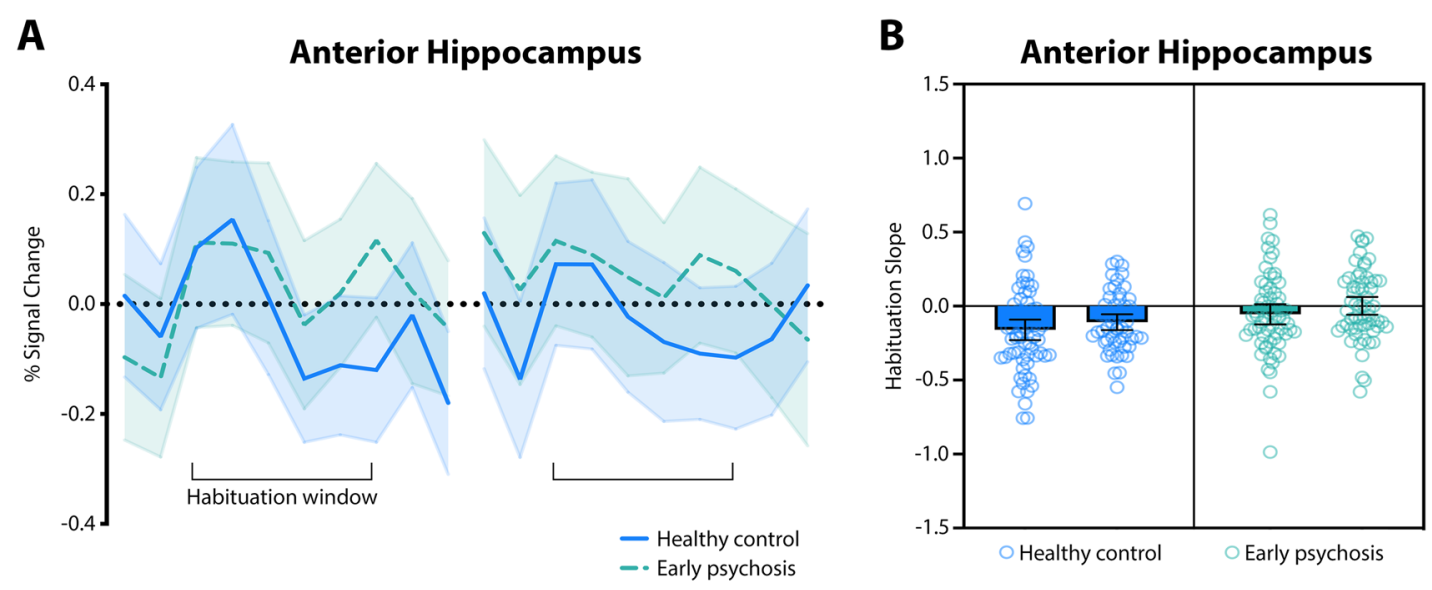

Occipital Pole
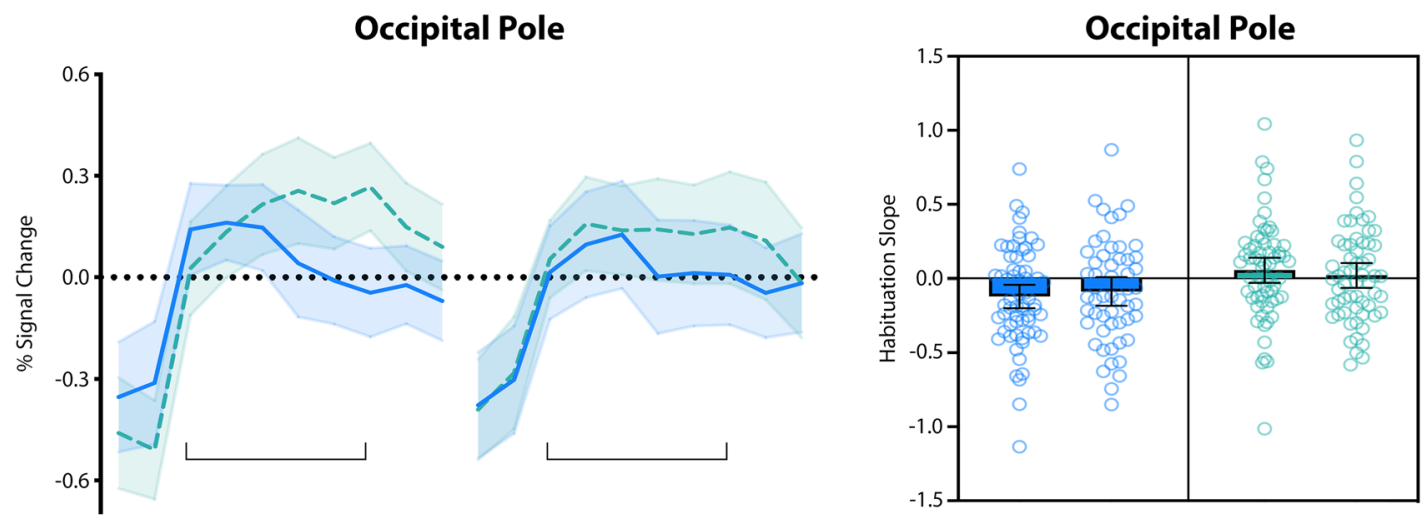

FFA
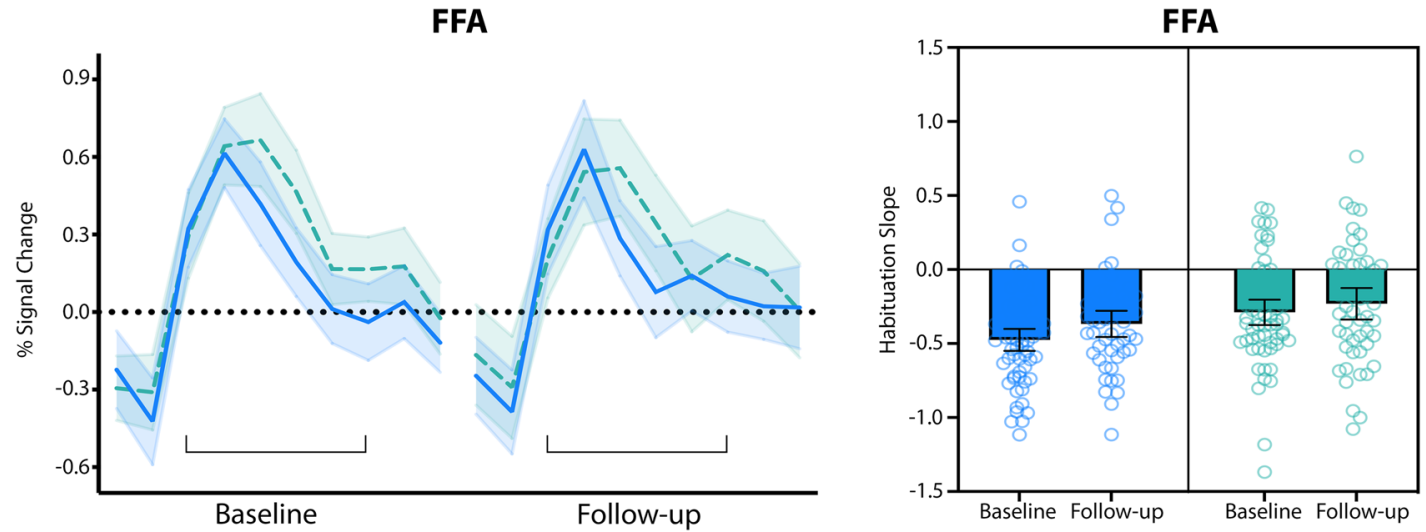

Fig. 2 Neural habituation to repeated objects at baseline and 2-year follow-up by group. Mean fMRI signal for the first $20 \mathrm{~s}$ of the repeated objects task is shown for each visit by ROI (A). Habituation slopes were calculated over a $10 \mathrm{~s}$ habituation window, with more negative slopes indicating greater habituation. The healthy control group showed negative habituation slopes across ROls (B). The early psychosis group showed negative habituation slopes in only the FFA, and habituation slopes in all ROls were smaller than in the healthy control group. Habituation slopes in both groups were consistent over time. Bars show the mean habituation slope by group (B); shaded areas (A); and error bars (B) indicate the $95 \%$ confidence interval.

showed a main effect of time ( $p s \geq 0.11)$ or a group by time interaction $(p s \geq 0.45)$, indicating habituation rates remained stable over time in both groups. Habituation rates did not differ between patients with a follow-up diagnosis of schizophrenia vs. schizophreniform disorder $(p s \geq 0.55)$. Group results were similar when examining habituation slopes uncorrected for novelty response (Supplementary Table 4).

We conducted follow-up one-sample $t$-tests to further characterize habituation within groups (Table 2). As expected, there was evidence of significant habituation in healthy control participants at both baseline and follow-up 
Table 2 Habituation.

\begin{tabular}{|c|c|c|c|c|c|c|c|c|}
\hline \multirow[t]{2}{*}{ Brain Region } & \multicolumn{4}{|l|}{ Baseline } & \multicolumn{4}{|l|}{ Follow-up } \\
\hline & $M \pm S D$ & $t$ & $d f$ & $p$ & $M \pm S D$ & $t$ & $d f$ & $p$ \\
\hline \multicolumn{9}{|l|}{ Healthy control } \\
\hline Anterior hippocampus & $-0.16 \pm 0.3$ & -4.63 & 66 & $<0.001^{*}$ & $-0.11 \pm 0.2$ & -3.97 & 51 & $<0.001^{*}$ \\
\hline Occipital pole & $-0.12 \pm 0.3$ & -3.02 & 66 & $0.002^{*}$ & $-0.09 \pm 0.4$ & -1.73 & 51 & $0.04^{*}$ \\
\hline FFA & $-0.48 \pm 0.3$ & -12.82 & 64 & $<0.001^{*}$ & $-0.37 \pm 0.3$ & -8.33 & 50 & $<0.001^{*}$ \\
\hline \multicolumn{9}{|l|}{ Early psychosis } \\
\hline Anterior hippocampus & $-0.06 \pm 0.3$ & -1.62 & 63 & 0.06 & $0.00 \pm 0.2$ & 0.09 & 58 & 0.54 \\
\hline Occipital pole & $0.06 \pm 0.3$ & 1.33 & 63 & 0.91 & $0.02 \pm 0.3$ & 0.56 & 58 & 0.71 \\
\hline FFA & $-0.29 \pm 0.3$ & -6.71 & 63 & $<0.001^{*}$ & $-0.23 \pm 0.4$ & -4.36 & 52 & $<0.001^{*}$ \\
\hline
\end{tabular}

Mean values \pm standard deviations are shown for each group. Asterisk $\left(^{*}\right)$ denotes significant $p$ values $(p \leq 0.05)$.

$M$ mean, SD standard deviation, FFA fusiform face area.

in all regions. In contrast, early psychosis patients showed evidence of habituation at both baseline and follow-up in only one region, the FFA. Although there was a trend for anterior hippocampal habituation at baseline in the early psychosis group $(p=0.06)$, there was no evidence of habituation at follow-up. There was no evidence of occipital pole habituation at either timepoint in early psychosis. Together, these findings suggest that habituation is significantly attenuated in the FFA and deficient in the anterior hippocampus and occipital pole in early psychosis compared to healthy controls, and these impairments are maintained over 2 years of illness.

\section{Clinical and cognitive correlates of habituation}

To determine whether individual variability in habituation rate was associated with function, we conducted correlations with clinical and cognitive measures. Habituation rates were not associated with general measures of cognition (SCIP) or verbal IQ (WTAR) in either group (Supplementary Table 5). There was a negative correlation between negative symptoms and habituation in the anterior hippocampus at follow-up $(r=-0.38$, FWEadjusted $p=0.03$ ). Negative symptoms were not correlated with baseline habituation in any region. Other clinical measures of psychosis, mood, duration of illness, and $\mathrm{CPZ}$ equivalent dose were not correlated with habituation at either visit.

\section{Discussion}

Our findings provide evidence for persistent habituation deficits in schizophrenia spectrum disorders (i.e., schizophreniform disorder, schizophrenia, schizoaffective disorder). In this study, we examined whether habituation deficits identified in a cohort of early psychosis patients at baseline within a visual processing network-the hippocampus, FFA, and occipital pole-progressed over two years of illness. Across regions, patients habituated less than healthy control participants. In contrast to our hypothesis that deficits would progress, we found that habituation deficits in psychosis patients were maintained, but did not worsen, over 2 years. Furthermore, while healthy control participants habituated rapidly in all brain regions, patients showed a mixture of slowed or failed habituation. Habituation in the FFA was slower in patients than in healthy control participants, but importantly, was achieved in both groups. In contrast, habituation in the anterior hippocampus and occipital pole was not detected in patients, indicating abnormally sustained brain activity in these regions, or habituation failure. Together, these findings provide evidence for a persistent habituation deficit maintained throughout the early stage of schizophrenia.

Hippocampal deficits are among the most consistent and replicable findings in schizophrenia ${ }^{63}$, have been demonstrated at the earliest stages of illness ${ }^{64,65}$, and have been linked with inhibitory/excitatory signaling imbalance originating in the anterior CA1 region during early illness ${ }^{63,65,66}$. Although hippocampal deficits are thought to progress throughout illness ${ }^{67,68}$, the timeline of this progression remains unclear as few longitudinal studies of the hippocampus have been conducted ${ }^{69}$. We previously found anterior hippocampal habituation deficits in both early psychosis ${ }^{17}$ and chronic schizophrenia ${ }^{16}$ in response to repeated visual stimuli, suggesting habituation deficits persist into later stages of illness. Our current longitudinal findings provide further support for this notion, and preliminary evidence that hippocampal inhibitory deficits may not progress in the early years of illness. These results are also consistent with recent findings in a partially-overlapping cohort, which showed hippocampal 
volume is reduced in the early stage of psychosis ${ }^{70}$ but does not further decline over a 2-year follow-up period ${ }^{71}$. Together, these findings suggest that hippocampal deficits are stable in the first 2 years of schizophrenia. However, follow-up longitudinal studies at longer intervals will be necessary to determine how long this window of stability may last.

Similarly, our findings in the occipital pole and FFA suggest that habituation deficits do not progress over the early course of illness. While sensory gating deficits in schizophrenia are well described in the auditory system ${ }^{72}$, fewer studies have focused on the visual system. Those that have suggest visual deficits in schizophrenia are driven by impairment in the dorsal ("where") visual stream, with relatively spared functioning in ventral ("what") visual stream ${ }^{72,73}$. However, other findings support functional deficits in the ventral visual stream that may be related to stimulus discrimination in schizophrenia ${ }^{74-76}$. The occipital pole, a region including both the superior and inferior posterior occipital cortex, is involved in processing macular (central) vision ${ }^{77}$. The hippocampus receives visual sensory input via the ventral visual stream, including direct connections from the occipital and fusiform cortex with reciprocal feedback from the hippocampus to visual cortex, which is thought to support visual discrimination and learning. Thus, paradigms that require visual learning, such as habituation paradigms, may be well-suited to elicit inhibitory deficits in the ventral visual pathway and hippocampus.

Although molecular studies suggest habituation can result from complex local mechanisms at the synapse ${ }^{78}$, network-level inhibitory potentiation may also drive habituation response ${ }^{79}$. Although we detected habituation deficits across regions, the current findings cannot clarify whether habituation deficits originate in one or all identified regions. The hippocampus may be uniquely situated to influence responses across the visual network ${ }^{80,81}$. In schizophrenia, there is compelling evidence for interneuron dysfunction in the hippocampus ${ }^{25}$, and reciprocal connections with the visual system may result in continued feedback to visual cortices. An influential early model of habituation ${ }^{82}$ proposed that as a stimulus is repeated, feedback inhibition promotes a decrease in neural response and a memory trace ${ }^{83}$. Hippocampal activity exemplifies this learning response-the hippocampus responds strongly to novelty ${ }^{84,85}$ but rapidly suppresses responses to repeated information via local interneuron-mediated inhibitory feedback ${ }^{86-89}$. Additionally, hippocampal habituation has been associated with visual-stimulus memory in healthy individuals and in schizophrenia ${ }^{16,17,90-92}$. However, given evidence for deficient inhibition within the visual cortex ${ }^{33,34}$ in schizophrenia, it is notable that both the hippocampus and occipital pole showed a similar failure to habituate. Future studies should consider habituation designs that could inform directional influences across regions.

Our results should be interpreted within the context of study limitations. The goal of this study was to examine habituation longitudinally in an a priori-defined set of visual processing and memory regions that showed habituation deficits in a prior cohort analysis ${ }^{17}$. Wholebrain approaches may reveal additional habituation deficits in schizophrenia. We used a visual-stimulus repetition paradigm, which can be readily conducted in an fMRI environment. However, as auditory system sensory gating deficits are well described in schizophrenia at the cellular and molecular level ${ }^{72}$, the translational utility of habituation paradigms may be increased by examining habituation to auditory stimuli. We used a habituation model that allowed us to characterize habituation rate independent of novelty response within regions of interest. Approaches that can be used at the voxel-wise level may reveal greater regional specificity of habituation deficits. Habituation may be influenced by a variety of factors, including attention, anxiety, arousal, and saccadic suppression $^{20,59,93,94}$. It is unlikely that our current findings are due to differences in attention, as target detection performance was high and did not differ between groups. However, future studies should consider measuring arousal, anxiety, and eye movements during the habituation session to account for potential influences.

In summary, we find evidence for persistent habituation deficits in schizophrenia. Over 2-year follow-up, early psychosis patients maintained slower or failed habituation across a set of regions including the hippocampus, occipital pole, and FFA. Although habituation is a ubiquitous and highly conserved process, individual differences have been reported in infancy ${ }^{95,96}$ and are hypothesized to fundamentally contribute to psychopathology, 6,9,97,98. Better understanding of translational mechanisms, such as habituation, are crucial in the exploration of novel interventions in schizophrenia ${ }^{99}$, and may be beneficial in understanding the mechanisms of onset and cognitive impairment associated with illness.

\section{Acknowledgements \\ This work was supported by the Charlotte and Donald Test Fund, NIMH grant R01-MH70560 (S.H.), the Vanderbilt Psychiatric Genotype/Phenotype Project, and the Vanderbilt Institute for Clinical and Translational Research (through grant 1-UL-1-TR000445 from the National Center for Research Resources/NIH).}

Conflict of interest

The authors declare that they have no conflict of interest.

Publisher's note

Springer Nature remains neutral with regard to jurisdictional claims in published maps and institutional affiliations.

Supplementary Information accompanies this paper at (https://doi.org/ 10.1038/s41398-020-01167-9). 
Received: 28 July 2020 Revised: 1 December 2020 Accepted: 9 December 2020

Published online: 05 January 2021

\section{References}

1. Thompson, R. F. \& Spencer, W. A. Habituation: a model phenomenon for the study of neuronal substrates of behavior. Psychol. Rev. 73, 16-43 (1966).

2. Poon, C. S. \& Young, D. L. Nonassociative learning as gated neural integrator and differentiator in stimulus-response pathways. Behav. Brain Funct. 2, 1-21 (2006).

3. Kavšek, M. Predicting later $\mathrm{lQ}$ from infant visual habituation and dishabituation: a meta-analysis. J. Appl. Dev. Psychol. 25, 369-393 (2004).

4. Yehuda, S., Shtrom, C. \& Peter, R. A possible link between intelligence level and habituation of the GSR. Int. J. Neurosci. 9, 53-55 (1979).

5. Freedman, R., Waldo, M., Bickford-Wimer, P. \& Nagamoto, H. Elementary neuronal dysfunctions in schizophrenia. Schizophr. Res. 4, 233-243 (1991).

6. Braff, D. L. \& Geyer, M. A. Sensorimotor gating and schizophrenia human and animal model studies. Arch. Gen. Psychiatry 47, 181-188 (1990).

7. Freedman, R. The Madness Within Us 1st edn. (Oxford University Press, New York, 2010).

8. Bleuler, E. Dementia Praecox or the Group of Schizophrenias (International Universities Press, New York, 1950).

9. Braff, D. L., Grillon, C. \& Geyer, M. A. Gating and habituation of the startle reflex in schizophrenic patients. Arch. Gen. Psychiatry 49, 206-215 (1992).

10. Bolino, F. et al. Sensorimotor gating and habituation evoked by electrocutaneous stimulation in schizophrenia. Biol. Psychiatry 36, 670-679 (1994).

11. Hollister, J. M., Mednick, S. A., Brennan, P. \& Cannon, T. D. Impaired autonomic nervous system habituation in those at genetic risk for schizophrenia. Arch. Gen. Psychiatry 51, 552-558 (1994).

12. Schiffer, R. A., Sigal, M. \& Mintz, M. Delayed habituation of the skinconductance orienting response correlates with impaired performance on the Wisconsin Card Sorting Task in schizophrenia. Psychiatry Res. 65, 107-112 (1996).

13. Olincy, A. et al. Inhibition of the P50 cerebral evoked response to repeated auditory stimuli: results from the Consortium on Genetics of Schizophrenia. Schizophr. Res. 119, 175-182 (2010).

14. Freedman, R. et al. Inhibitory gating of an evoked response to repeated auditory stimuli in schizophrenic and normal subjects. Human recordings, computer simulation, and an animal model. Arch. Gen. Psychiatry 53, 1114-1121 (1996).

15. Grunwald, T. et al. Neuronal substrates of sensory gating within the human brain. Biol. Psychiatry 53, 511-519 (2003).

16. Williams, L. E., Blackford, J. U., Luksik, A., Gauthier, I. \& Heckers, S. Reduced habituation in patients with schizophrenia. Schizophr. Res. 151, 124-132 (2013).

17. Avery, S. N. et al. Disrupted habituation in the early stage of psychosis. Biol. Psychiatry Cogn. Neurosci. Neuroimaging 4, 1004-1012 (2019).

18. Holt, D. J. et al. Sustained activation of the hippocampus in response to fearful faces in schizophrenia. Biol. Psychiatry 57, 1011-1019 (2005).

19. Ramaswami, M. Network plasticity in adaptive filtering and behavioral habituation. Neuron 82, 1216-1229 (2014).

20. Groves, P. M. \& Thompson, R. F. Habituation: a dual-process theory. Psychol. Rev. 77, 419-450 (1970).

21. Glanzman, D. L. Olfactory habituation: fresh insights from flies. Proc. Natl Acad. Sci. USA 108, 14711-14712 (2011).

22. Das, S. et al. Plasticity of local GABAergic interneurons drives olfactory habituation. Proc. Natl Acad. Sci. USA 108, 2-10 (2011).

23. Gonzalez-Burgos, G., Cho, R. Y. \& Lewis, D. A. Alterations in cortical network oscillations and parvalbumin neurons in schizophrenia. Biol. Psychiatry 77, 1031-1040 (2015).

24. McHugo, M. et al. Hyperactivity and reduced activation of anterior hippocampus in early psychosis. Am. J. Psychiatry 176, 1030-1038 (2019).

25. Heckers, S. \& Konradi, C. GABAergic mechanisms of hippocampal hyperactivity in schizophrenia. Schizophr. Res. 167, 4-11 (2015).

26. Benes, F. M. et al. Regulation of the GABA cell phenotype in hippocampus of schizophrenics and bipolars. Proc. Natl Acad. Sci. USA 104, 10164-10169 (2007)

27. Yamaguchi, S., Hale, L. A., D'Esposito, M. \& Knight, R. T. Rapid prefrontalhippocampal habituation to novel events. J. Neurosci. 24, 5356-5363 (2004).

28. Breiter, H. C. et al. Response and habituation of the human amygdala during visual processing of facial expression. Neuron 17, 875-887 (1996).
29. Fischer, H. et al. Brain habituation during repeated exposure to fearful and neutral faces: a functional MRI study. Brain Res. Bull. 59, 387-392 (2003).

30. Blackford, J. U., Allen, A. H., Cowan, R. L. \& Avery, S. N. Amygdala and hippocampus fail to habituate to faces in individuals with an inhibited temperament. Soc. Cogn. Affect Neurosci. 8, 143-150 (2013).

31. Kim, K., Hsieh, L.-T., Parvizi, J. \& Ranganath, C. Neural repetition suppression effects in the human hippocampus. Neurobiol. Learn. Mem. 173, 107269 https://doi.org/10.1016/j.nlm.2020.107269 (2020).

32. McGarrity, S., Mason, R., Fone, K. C., Pezze, M. \& Bast, T. Hippocampal neural disinhibition causes attentional and memory deficits. Cereb. Cortex 27 4447-4462 (2017).

33. Yoon, J. H. et al. Reduced in vivo visual cortex GABA in schizophrenia, a replication in a recent onset sample. Schizophr. Res. 215, 217-222 (2020).

34. Yoon, J. H. et al. GABA concentration is reduced in visual cortex in schizophrenia and correlates with orientation-specific surround suppression. J. Neurosci. 30, 3777-3781 (2010)

35. Weigelt, S., Muckli, L. \& Kohler, A. Functional magnetic resonance adaptation in visual neuroscience. Rev. Neurosci. https://doi.org/ 10.1515/REVNEURO.2008.19.4-5.363 (2008).

36. Summerfield, C., Trittschuh, E. H., Monti, J. M., Mesulam, M. M. \& Egner, T. Neural repetition suppression reflects fulfilled perceptual expectations. Nat. Neurosci. 11, 1004-1006 (2008)

37. Woo, T. U. W. Neurobiology of schizophrenia onset. Curr. Top Behav. Neurosci. 16, 267-295 (2014).

38. Birchwood, M., Todd, P. \& Jackson, C. Early intervention in psychosis. The critical period hypothesis. Br. J. Psychiatry Suppl. 172, 53-59 (1998).

39. Pinsker, H., Kupfermann, I., Castellucci, V. \& Kandel, E. Habituation and dishabituation of the gill-withdrawal reflex in Aplysia. Science 167, 1740-1742 (1970).

40. First, M., Spitzer, R. R. L., Gibbon, M. \& Williams, J. Structured Clinical Interview for the DSM-IV-TR Axis I Disorders, Research Version, Patient Edition (SCID-I/P). (Biometrics Research, New York State Psychiatric Institute, New York, 2002).

41. American Psychiatric Association. Diagnostic and statistical manual of mental disorders. 5th edn. (American Psychiatric Association, Washington, 2013).

42. Avery, S. N. et al. Relational memory in the early stage of psychosis: a 2-year follow-up study. Schizophr. Bull. 1-12 https://doi.org/10.1093/schbul/sbaa081 (2020).

43. First, M. B., Gibbon, R. L., Spitzer, R. L. \& Williams, J. B. W. User's Guide for the SCID-I: Structured Clincial Interview for DSM-IV Axis I Disorders. (New York State Psychiatric Institute, 1995).

44. Kay, S. R. et al. The positive and negative syndrome scale (PANSS) for schizophrenia. Schizophr. Bull. 13, 261-276 (1987).

45. Hamilton, M. A rating scale for depression. J. Neurol. Neurosurg. Psychiatry 23 56-62 (1960).

46. Young, R. C., Biggs, J. T., Ziegler, V. E. \& Meyer, D. A. A rating scale for mania: reliability, validity and sensitivity. Br. J. Psychiatry 133, 429-435 (1978).

47. Purdon, S. E. The Screen for Cognitive Impairment in Psychiatry (SCIP): Administration Manual and Normative Data. (PNL Inc, Edmonton, 2005).

48. Friston, K. J. et al. Statistical parametric maps in functional imaging: a general linear approach. Hum. Brain Mapp. 2, 189-210 (1994).

49. Asman, A. J., \& Landman, B. A. Characterizing spatially varying performance to improve multi-atlas multi-label segmentation. Inf. Process Med. Imaging. 22, 85-96 https://doi.org/10.1007/978-3-642-22092-0_8 (2011).

50. Asman, A. J., Dagley, A. S. \& Landman, B. A. Statistical label fusion with hierarchical performance models. Med. Imaging 2014 Image Process. 9034, 90341E https://doi.org/10.1117/12.2043182 (2014).

51. Poppenk, J., Evensmoen, H. R., Moscovitch, M. \& Nadel, L. Long-axis specialization of the human hippocampus. Trends Cogn. Sci. 17, 230-240 (2013).

52. Strange, B. A., Witter, M. P., Lein, E. S. \& Moser, E. I. Functional organization of the hippocampal longitudinal axis. Nat. Rev. Neurosci. 15, 655-669 (2014).

53. Wong, Y. K. \& Gauthier, I. A multimodal neural network recruited by expertise with musical notation. J. Cogn. Neurosci. 22, 695-713 (2010).

54. Tarr, M. J. \& Gauthier, I. FFA: a flexible fusiform area for subordinate-level visual processing automatized by expertise. Nat. Neurosci. 3, 764-769 (2000).

55. McGugin, R. W., Van Gulick, A. E., Tamber-Rosenau, B. J., Ross, D. A. \& Gauthier, I. Expertise effects in face-selective areas are robust to clutter and diverted attention, but not to competition. Cereb. Cortex 25, 2610-2622 (2015).

56. Grill-Spector, K., Henson, R. \& Martin, A. Repetition and the brain: neural models of stimulus-specific effects. Trends Cogn. Sci. 10, 14-23 (2006). 
57. Rankin, C. H. et al. Habituation revisited: an updated and revised description of the behavioral characteristics of habituation. Neurobiol. Learn Mem. 92, 135-138 (2009).

58. Brett, M., Anton, J.-L. L., Valabregue, R. \& Poline, J.-B. Region of interest analysis using an SPM toolbox [abstract] Presented at the 8th International Conference on Functional Mapping of the Human Brain, June 2-6, 2002, Sendai, Japan. Neuroimage 16, 497 (2002).

59. Avery, S. N. \& Blackford, J. U. Slow to warm up: the role of habituation in social fear. Soc. Cogn. Affect Neurosci. 11, 1832-1840 (2016).

60. Montagu, J. D. Habituation of the psycho-galvanic reflex during serial tests. J. Psychosom. Res. 7, 199-214 (1963).

61. Plichta, M. M. et al. Amygdala habituation: a reliable fMRI phenotype. Neuroimage 103, 383-390 (2014).

62. Vandekar, S., Tao, R. \& Blume, J. A robust effect size index. Psychometrika $\mathbf{8 5}$, 232-246 (2020).

63. Heckers, S. \& Konradi, C. Hippocampal neurons in schizophrenia. J. Neural Transm. 109, 891-905 (2002)

64. Pantelis, C. et al. Neurobiological markers of illness onset in psychosis and schizophrenia: the search for a moving target. Neuropsychol. Rev. 19, 385-398 (2009).

65. Lieberman, J. A. et al. Hippocampal dysfunction in the pathophysiology of schizophrenia: a selective review and hypothesis for early detection and intervention. Mol. Psychiatry 23, 1764-1772 (2018).

66. Schobel, S. A. et al. Imaging patients with psychosis and a mouse model establishes a spreading pattern of hippocampal dysfunction and implicates glutamate as a driver. Neuron 78, 81-93 (2013).

67. Velakoulis, D. et al. Hippocampal volume in first-episode psychoses and chronic schizophrenia: a high-resolution magnetic resonance imaging study. Arch. Gen. Psychiatry 56, 133-140 (1999).

68. Velakoulis, D., Wood, S. J., Mcgorry, P. D. \& Pantelis, C. Evidence for progression of brain structural abnormalities in schizophrenia: beyond the neurodevelopmental model. Aust. N. Z. J. Psychiatry 34, S113-S126. (2000).

69. Heilbronner, U., Samara, M., Leucht, S., Falkai, P. \& Schulze, T. G. The longitudinal course of schizophrenia across the lifespan: clinical, cognitive, and neurobiological aspects. Harv. Rev. Psychiatry 24, 118-128 (2016).

70. McHugo, M. et al. Regionally specific volume deficits along the hippocampal long axis in early and chronic psychosis. Neurolmage Clin. 20, 1106-1114 (2018).

71. McHugo, M. et al. Hippocampal volume in early psychosis: a 2-year longitudinal study. Transl. Psychiatry 10, 306 (2020).

72. Javitt, D. C. \& Freedman, R. Sensory processing dysfunction in the personal experience and neuronal machinery of schizophrenia. Am. J. Psychiatry 172 17-31 (2015)

73. Javitt, D. C. Sensory processing in schizophrenia: neither simple nor intact. Schizophr. Bull. 35, 1059-1064 (2009).

74. Maher, S. et al. Functional disconnection between the visual cortex and right fusiform face area in schizophrenia. Schizophr. Res. 209, 72-79 (2019).

75. Plomp, G. et al. Electrophysiological evidence for ventral stream deficits in schizophrenia patients. Schizophr. Bull. 39, 547-554 (2013).

76. Maher, S., Ekstrom, T., Holt, D., Ongur, D. \& Chen, Y. The core brain region for face processing in schizophrenia lacks face selectivity. Schizophr. Bull. 42, 666-674 (2016).

77. Isa, K., Miyashita, K., Yanagimoto, S., Nagatsuka, K. \& Naritomi, H. Homonymous defect of macular vision in ischemic stroke. Eur. Neurol. 46, 126-130 (2001).

78. McDiarmid, T. A., Yu, A. J. \& Rankin, C. H. Habituation is more than learning to ignore: multiple mechanisms serve to facilitate shifts in behavioral strategy. BioEssays 41, 1-10. (2019).
79. Thompson, R. F. Habituation: a history. Neurobiol. Learn Mem. 92, 127-34 (2009).

80. Haggerty, D. C. \& Ji, D. Activities of visual cortical and hippocampal neurons co-fluctuate in freely moving rats during spatial behavior. elife 4, 1-23 (2015).

81. Turk-Browne, N. B. The hippocampus as a visual area organized by space and time: a spatiotemporal similarity hypothesis. Vis. Res. 165, 123-130 (2019).

82. Sokolov, E. N. Higher nervous functions; the orienting reflex. Annu. Rev. Physiol. 25, 545-580 (1963).

83. Barron, H. C., Vogels, T. P., Behrens, T. E. \& Ramaswami, M. Inhibitory engrams in perception and memory. Proc. Natl Acad. Sci. USA 114 6666-6674 (2017).

84. Fried, I., MacDonald, K. A. \& Wilson, C. L. Single neuron activity in human hippocampus and amygdala during recognition of faces and objects. Neuron 18, 753-765 (1997).

85. Kreiman, G., Koch, C. \& Fried, I. Category-specific visual responses of single neurons in the human medial temporal lobe. Nat. Neurosci. 3, 946-953 (2000).

86. Arriaga, M. \& Han, E. B. Dedicated hippocampal inhibitory networks for locomotion and immobility. J. Neurosci. 37, 9222-9238 (2017).

87. Wilson, F. A. W., Rolls, E. T. \& Wilson, F. A. W. The effects of stimulus novelty and familiarity on neuronal activity in the amygdala of monkeys performing recognition memory tasks. Exp. Brain Res. 93, 367-382 (1993).

88. Booker, S. A. \& Vida, I. Morphological diversity and connectivity of hippocampal interneurons. Cell Tissue Res. 373, 619-641 (2018).

89. Murty, V. P., Ballard, I. C., MacDuffie, K. E., Krebs, R. M. \& Adcock, R. A. Hippocampal networks habituate as novelty accumulates. Learn Mem. 20, 229-235 (2013).

90. Avery, S. N. et al. Habituation during encoding: a new approach to the evaluation of memory deficits in schizophrenia. Schizophr. Res. 223, 179-185 (2020).

91. Brady, R. J., Basile, B. M. \& Hampton, R. R. Hippocampal damage attenuates habituation to videos in monkeys. Hippocampus 29, 1121-1126 (2019).

92. Bunzeck, N., Guitart-Masip, M., Dolan, R. J. \& Duzel, E. Pharmacological dissociation of novelty responses in the human brain. Cereb. Cortex $\mathbf{2 4}$ 1351-1360 (2013)

93. Patacchioli, F. R., Taglialatela, G., Angelucci, L., Cerbone, A. \& Sadile, A. G. Adrenocorticoid receptor binding in the rat hippocampus: strain-dependent covariations with arousal and habituation to novelty. Behav. Brain Res. 33, 287-300 (1989).

94. Berman, R. A., Cavanaugh, J., McAlonan, K. \& Wurtz, R. H. Neural circuits: a circuit for saccadic suppression in the primate brain. J. Neurophysiol. 117, 1720 (2017).

95. Bushnell, I. W. R. Discrimination of faces by young infants. J. Exp. Child Psychol. 33, 298-308 (1982).

96. Snyder, K. A. \& Keil, A. Repetition suppression of induced gamma activity predicts enhanced orienting toward a novel stimulus in 6-month-old infants. J. Cogn. Neurosci. 20, 2137-2152 (2008).

97. Davidson, R. J. \& Irwin, W. The functional neuroanatomy of emotion and affective style. Trends Cogn. Sci. 3, 11-21 (1999).

98. McDiarmid, T. A., Bernardos, A. C. \& Rankin, C. H. Habituation is altered in neuropsychiatric disorders-a comprehensive review with recommendations for experimental design and analysis. Neurosci. Biobehav. Rev. 80, 286-305 (2017).

99. Barron, H. C., Garvert, M. M. \& Behrens, T. E. J. Repetition suppression: a means to index neural representations using BOLD? Philos. Trans. R. Soc. B. Biol. Sci. https://doi.org/10.1098/rstb.2015.0355 (2016). 